\title{
CHANGES OF VIBRATIONAL LIFETIMES WITH MINOR STRUCTURAL MODIFICATION OF SMALL POLYATOMIC MOLECULES
}

\author{
C. KOLMEDER, W. ZINTH and W. KAISER \\ Physik Department der Techmischen Umu'ersitöt ATunchcn, Mfumch, Gerniany'
}

Reccived 7 July 1982

Substantual changes of population lifetumes of $\mathrm{CH}-s t$ retciung modes are observed when two atoms are exchanged in $\mathrm{CH}_{2}=\mathrm{CCl}_{2}$ to form trans $\mathrm{CHCl}=\mathrm{CHCl}$ and when three deuterons are substututed in $\mathrm{C}_{6} \mathrm{H}_{6}$ to form $1,3,5-\mathrm{C}_{6} \mathrm{H}_{3} \mathrm{D}_{3}$ The measured lifetumes are in good agsecment with estimates based on I"ermu resonance-nixing whel is inferred Ironi int rured and Raman spect ta

\section{Introduction}

With the help of ultrashort laser pulses it has been possible to measure durectly the population lifetime of well-defined vibrational modes of polyatomc molecules in the liquid state. For a number of nolecules the population decay was found to be of the order of picoseconds [1-4]. Spectal attention was paud to $\mathrm{CH}$-stretching modes which are of considerable $\mathrm{im}$ portance for the radiationless decay of electronsc excitations.

The investigation of small molecules is faclitated by the small number of normal modes [5]. Frequently we know quite accurately the intially prepared states and have strong indications as to the final states. Recently, It became apparent that mixing by Fermi resonance represents a major coupling mechanism between the intial and the final states, 1.e. intramolecular anharmonic coupling between neighboring states of like symmetry is of major importance for the lifetime of the vibrational states [2].

In this paper we present new expenmental evidence of the inportance of Fermu resonance for the lifetimes of $\mathrm{CH}$-stretchung modes. Small changes in the molecular structure can give rise to large changes of Fermi resonance muxing which in turn has a profound effect on the population lifetime. As examples we show data on $\mathrm{CH}_{2}=\mathrm{CCl}_{2}$ and trans $\mathrm{CHCl}=\mathrm{CHCl}$ where only two atoms exchange therr positions while the basic structure and the number of atoms are preserved. In addition, we compare the lifetumes of the $\mathrm{CH}$ modes of benzene $\mathrm{C}_{6} \mathrm{H}_{6}$ with the partially deuterated form $1,3,5-C_{6} H_{3} D_{3}$.

\section{Experimental}

In our investigations vibrational modes are resonantly excited by ultrashort infrared pulses and the unstantaneous degree of population is montored by spontaneous antr-Stokes Raman scattering of a second probe pulse. Thus two ultrashort light pulses of widely dufferent frequencies are required in our experiments.

The essential features of our experimental system have been described [4]. Briefly, a Nd-giass oscillator amplifier provides single ultrashort laser pulses at $9495 \mathrm{~cm}^{-1}$. These pulses serve as a pump for the parametric generation of infrared pulses tunable between 2800 and $3200 \mathrm{~cm}^{-1}$. The parametric system consists of three $\mathrm{LiNbO}_{3}$ crystals mounted on highprecision turntables. The IR pulses have a duration of 3 ps and $\approx 10^{15}$ quanta per pulse. Probe pulses - at the second-harmonic frequency of the laser pulses, i.e. at $18990 \mathrm{~cm}^{-1}$ - monitor the degree of vibrational excitation as a function of tume. A large fraction of the generated anti-Stokes photons are collected by placing the specimen at one focus of an ellipsoi- 
dal murror and focusing the radiation on the entrance slit of a spectrometer of large aperture (spectral resolution $\approx 80 \mathrm{~cm}^{-1}$ ). The exciting IR pulses and the green probing pulses have perpendıcular polarisation. The zero point of the delay tume and the time resolu. tion of the system were determined from the crosscorrelation curve between IR pulse and probe pulse. To this end the sum frequency of the two uncident pulses produced with the help of a properly oriented non-linear $\mathrm{LiNbO}_{3}$ crystal is measured sumultaneously as a function of the delay tume between the two pulses.

Infrared and Raman spectra of figs. 1 and 3 were taken with standard instrumentation of spectral resolution 4 and $2 \mathrm{~cm}^{-1}$, respectively.

\section{Results and discussion}

Recent investıgations have shown that there is a very rapid exchange of vibrational energy between structurally simular and energetically neighboring vibrational states. For instance, vibrational energy is quickly transferred from the asymmetric to the symmetric $\mathrm{CH}_{2}$-stretching mode in $\mathrm{CH}_{2} \mathrm{Cl}_{2}$ or from an infrared active to a Raman active $\mathrm{CH}$-stretching mode in benzene $[2,3]$. On account of this rapid energy exchange in $<1$ ps several vibrational states $N$ are populated in certain molecules during the excitation pulse. When several vibrational states are pumped in the molecule one may observe a longer decay of the reservour of states.

Theoretical considerations show that the energy transfer between $\mathrm{CH}$-stretching modes of essentially the same energy occurs in a time approxumately equal to the dephasing tıme $T_{2}$. If the energy difference $\hbar \omega$ $\therefore \hbar \omega_{1}-\hbar \omega_{\mathrm{f}}$ is substantial we need a correction factor,

$T_{1}\left(\omega_{1} \rightarrow \omega_{f}\right)=T_{2}\left(\omega_{1}\right) \exp \left[(\omega / \Omega)^{2 / 3}\right]$,

where $\Omega$ is $100 \mathrm{~cm}^{-1}$ We recall that the width of the Raman line gives a lower limit on the pure dephasing time since other broadenung processes may contribute to the lunewidth.

As pointed out above, the anharmonic coupling, the Fermi resonance, between different but neighborung energy states leads to an intramolecular decay of vibrational energy. For instance, energy flows from the $\mathrm{CH}$-stretching modes via overtones and combination modes to lower-energy states. The degree of Fermi resonance manufests itself in the infrared and Raman spectra. Overtones and higher-order combination modes borrow intensity from $\mathrm{CH}$-stretching modes. We define as a measure of Fermi resonancemixing the intensity ratio $R$ between the final and inttial state taken from the infrared or Raman spectrum [6]. In a recent publication a formula was derived which allows us to estumate the lifetime $T_{1}$ of vibrational states [2]:

$T_{1}=N(1+R)^{2} R^{-1} \exp \left[(\omega / \Omega)^{2 / 3}\right] T_{2}(\mathrm{f})$.

$N$ corresponds to the number of states initially excited. $R$ is a measure of the Fermi resonance, and $T_{2}(f)$ stands for the dephasing time of the final state. $T_{2}(f)$ may be est imated from the Raman linewidth $\Delta \nu$ as $T_{2}(f)=(2 \pi c \Delta \nu)^{-1}$. (It should be noted that $T_{2}(f)$ is equivalent to $\frac{1}{2} T_{2}$ measured in coherent Raman scattering.) The energy $7 w$ represents the difference between the intial and the final state. $\hbar \Omega$ has a value close to $100 \mathrm{~cm}^{-1}$. Difficulties arise for very strong vibrational coupling. The Fermi resonance interaction may become so strong that the theory no longer holds, It should be mentioned that the determination of $T_{2}(\eta)$ is difficult in cases of complex spectra. A modified [6] equation (2) may be used where $T_{2}(\mathrm{i})$, the dephasing time of the intial state, is introduced.

In this paper we present data on four molecules.

\section{I. $\mathrm{CH}_{2}=\mathrm{CCl}_{2}$}

In figs. $1 \mathrm{a}$ and $1 \mathrm{~b}$ we see the symmetric $\left(\nu_{1}\right)$ and asymmetric $\left(v_{7}\right) \mathrm{CH}_{2}$-stretching vibrations separated by $100 \mathrm{~cm}^{-1}$. The $\nu_{1}$ mode 15 excited and its decay is monitored. According to eq. (1) we estimate an energy-transfer tume between the two $\mathrm{CH}$-stretching modes of $T_{1}\left(\nu_{1} \rightarrow \nu_{7}\right) \approx 3.3 \mathrm{ps}$, where $T_{2}(\mathrm{f}) \approx 1.2 \mathrm{ps}$ was taken from the Raman spectrum.

Fig. 1a shows strong Fermi resonance between $\nu_{1}$ and $\nu_{2}+\nu_{3}$, both of $\mathrm{A}_{1}$ symmetry, and between $\nu_{7}$ and $\nu_{2}+\nu_{6}+\nu_{11}$, both of $B_{1}$ symmetry [7]. This observation suggests that we have to consider two decay channels. For the decay $\nu_{1} \rightarrow \nu_{2}+\nu_{3}$ we estimate the intensity ratio $R=0.2 \pm 0.05$ and calculate $T_{2}$ (f) $=0.3 \mathrm{ps}$ from the Raman linewidth of $\Delta \tilde{\nu}=17 \mathrm{~cm}^{-1}$. With $N=1$ and $\omega=45 \mathrm{~cm}^{-1}$, we calculate from eq. (2) a value of $T_{1}=4 \pm 2$ ps. 

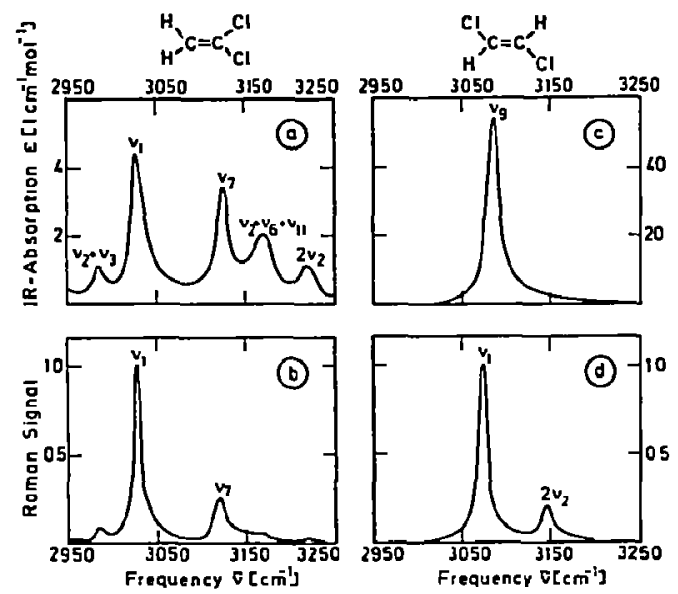

Fig. 1. Infrared absorption (a) and Raman (b) spectra of $\mathrm{CH}_{2} \mathrm{CCl}_{2}$ between 2950 and $3250 \mathrm{~cm}^{-1}$. Two combunition tones are in strong Termi resonance with the two $\mathrm{CH}$-stretcliung modes at $\nu_{1}$ and $\nu_{7}$ Infrared absorption (c) and Raman (d) spectra of trans-CHCiCHCl There is less Гermi resonance mixing than in $\mathrm{CH}_{2} \mathrm{CCl}_{2}$.

For the second decay channel $\nu_{7} \rightarrow \nu_{2}+\nu_{6}+\nu_{11}$ we have to take a short dephasing time $\bar{T}_{2}$ (f) of the combination tone. We should remember that every component of $\nu_{2}+\nu_{6}+\nu_{11}$ contributes to the dephasing and, in addition, the low-frequency $\mathrm{CH}$ modes undergo faster dephasing than the $\mathrm{CH}$-stretching modes. We estimate $T_{2}\left(\nu_{2}+\nu_{6}+\nu_{11}\right) \approx 02$ ps which is consistent with the observed linewidth. With $R=$ $0.6 \pm 0.1, N=1$, and $\omega=45 \mathrm{~cm}^{-1}$ we calculate $T_{1}=$ 1.5 ps.

The estımates given above indicate that vibrational energy flows faster out of the $\nu_{7}$ mode than in the energy transfer $\nu_{1} \rightarrow \nu_{7}$. For the interrogated $\nu_{1}$ mode we simply add the two decay channels $\nu_{1} \rightarrow \nu_{7}$ and $\nu_{1} \rightarrow \nu_{2}+\nu_{3}$ and arrive at a value of $T_{1}\left(\nu_{1}\right) \approx 2 \mathrm{ps}$.

In fig. $2 \mathrm{a}$ we present experimental data. The $v_{1}$ mode at $3036 \mathrm{~cm}^{-1}$ is resonantly excited by an infrared puise and the antistokes scattering of the same $\nu_{1}$ mode is observed. The scattered Raman signal rises to a slightly delayed maximum during the excitation process and decays whth a relaxation time $T_{1}=3 \pm 1$ ps. This expenmental finding is in fair agreement with the calculated value for $T_{1}$ given above. The broken curves in fig. 2 correspond to the simultaneously measured cross-correlation curves.

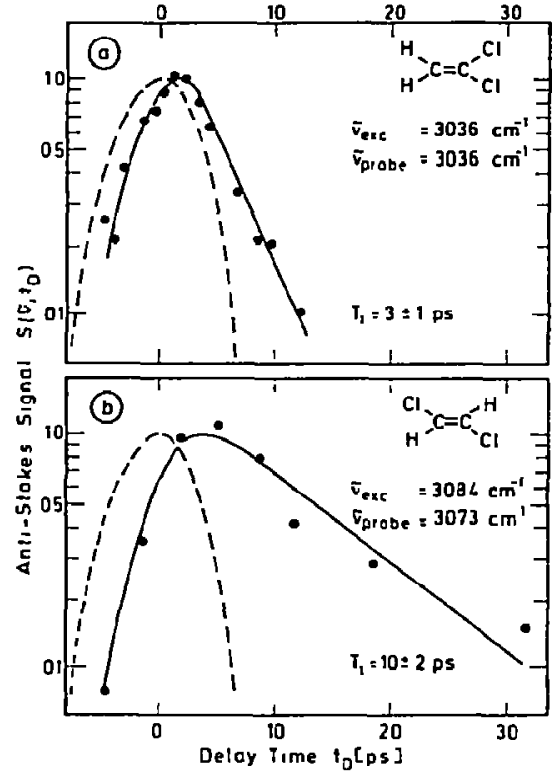

Гig 2 Anti-Stokes scatterung signal versus delay tume of the probung pulse (a) $\mathrm{CH}_{2} \mathrm{CCl}_{2}$ in $\mathrm{CCl}_{4}(c=035 \mathrm{~m} \mathrm{f}$ ) The decay of the $\mathrm{CH}_{2}$-stretching mode at $3036 \mathrm{~cm}^{-1}$ is shown (b) trans-CHClCHCl in $\mathrm{CCl}_{4}(c=035 \mathrm{~m}$.f $)$ The Cll-stretclung mode at $3084 \mathrm{~cm}^{-1}$ is excuted and the mode at $3073 \mathrm{~cm}^{-1}$ is montiored The broken curves are the cross-correlstion tunetions of the IR csciting and green probing pulses

\subsection{Trans- $\mathrm{CHCl}=\mathrm{CHCl}$}

In fig. Ic wc see the infrared-active CH-stretchung mode $\nu_{\mathbf{9}}$ and in fig. $1 \mathrm{~d}$ the Raman-active symmetric $\nu_{1}$ mode. Contrary to $\mathrm{CH}_{2} \mathrm{CCl}_{2}$ discussed above, we find a considerably smaller Fernu resonance in trans$\mathrm{CHCl}=\mathrm{CHCl}$. The Raman spectrum of fig. 1d suggests some Fermi resonance between $\nu_{1}$ and $2 \nu_{2}$, both of $A_{g}$ symmetry $[5,7]$. With the values $R=0.15 \pm 002$, $T_{2}=0.3 \mathrm{ps}, N=2$, and $\omega=79 \mathrm{~cm}^{-1}$ we calculate $T_{1}$ $=13$ ps. It should be noted that there possibly are additional weak Fermi resonances between the $\nu_{9}$ mode and higher combination modes (eg. $\nu_{2}+\nu_{5}$ $+\nu_{10}$ ) buried under the high-frequency tal of the $\nu_{9}$ fundamental. These additional decay channels may reduce somewhat the estumated $T_{\mathrm{L}}$ value.

The tume dependence of the $\mathrm{CH}$-stretching modes is depicted in fig. $2 \mathrm{~b}$. The molecule is excited via the $v_{9}$ mode at $3084 \mathrm{~cm}^{-1}$ and the population of the $\nu_{1}$ mode at $3073 \mathrm{~cm}^{-1}$ is monitored. The decay of the 
signal curve gaves a long lifetime of the two $\mathrm{CH}$-stretching modes of $T_{1}=10 \pm 2 \mathrm{ps}$. Thus number is in good agreement with the value estımated above. The small untramolecular coupling gives nse to the longer vibratoonal lifetime

\section{3. $\mathrm{C}_{6} H_{6}$}

Benzene lias been discussed in a recent publication [3]. For clanty and ready comparison we repeat some of the previous results. In fig. 3 a the infrared absorption spectrum shows strong Ferm resonance between the $\nu_{20}$ fundamental $\mathrm{CH}$-stretchung mode and several double and triple combination modes [3,8]. A nore detailed inspection of the vibratiunal energy states of benzene suggests numerous decay channels between the $\mathrm{CH}$-stretching modes and several lower vibrational modes of the molecule It is not surprising, therefore, that we undeed find a very short lifetime of the energy in the $\mathrm{CH}$-stretching modes In fig. $4 \mathrm{a}$ the benzene sample was excited va a combunation mode at 3090 $\mathrm{cm}^{-1}$. We see the data points in fig. $4 \mathrm{a}$ close to the time resolution of the system (the broken curve is the cross-correlation curve) and estimate a decay time of $T_{\mathrm{l}}=1 \pm 0.5 \mathrm{ps}$.
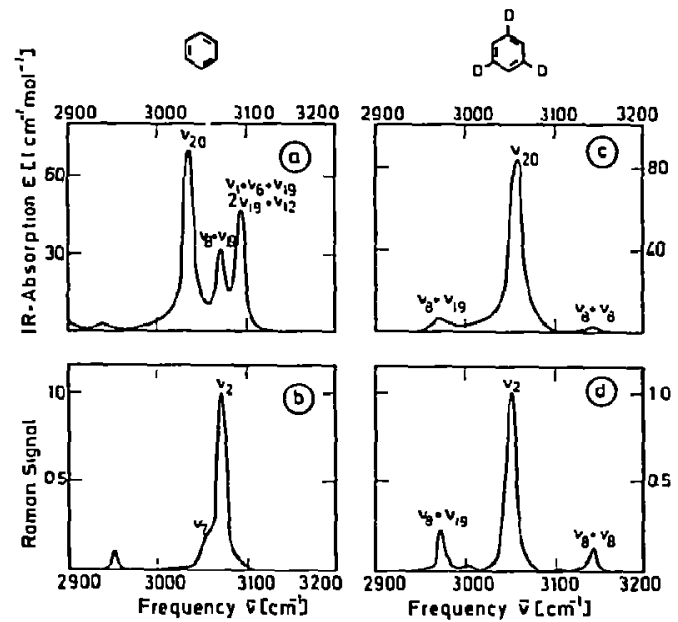

rig 3. Infrared absorption (a) and Raman (b) spectra of benzcne around $3050 \mathrm{~cm}^{-1}$ Note the strong anharmonic interacton with combunation modes. Infrared absorption (c) and Raman (d) spectra of $1,3,5 d_{3}$-benzenc. The Fermu resonance with combination modes is smaller than in $\mathrm{C}_{6} \mathrm{H}_{6}$ (a)

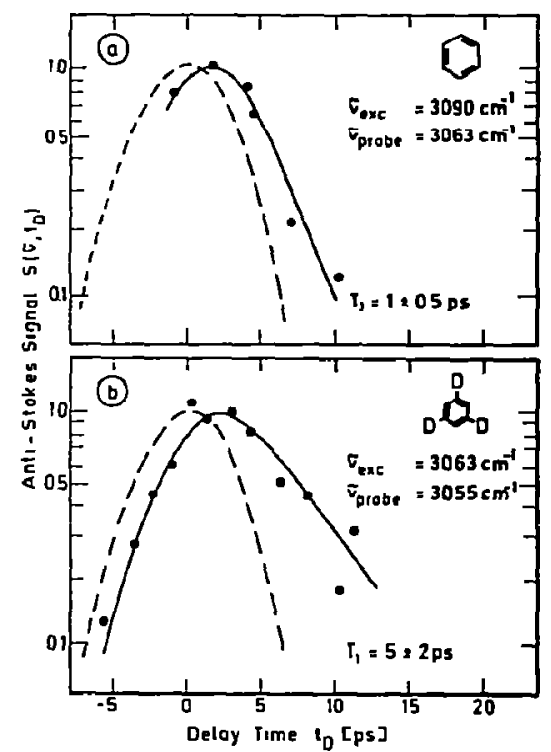

$\Gamma_{4 .} 4$ Anti-Stohes scattering signal versus tume. (a) The $\mathrm{CH}$ stretching modes of neat benzene. (b) The $\mathrm{CH}$-stretching modes of neat $1,3,5-d_{3}$-benzene The lifetime of the $\mathrm{CH}$ stretchung modes uncreases upon dcuteration.

\section{$3.41,3,5-C_{6} H_{3} D_{3}$}

The substitution of three hydrogen atoms by three deuterons changes the infrared and Raman spectra substantially. Comparing the infrared spectra of figs. $3 \mathrm{a}$ and $3 \mathrm{c}$ shows less Fermi resonances in $\mathrm{C}_{6} \mathrm{H}_{3} \mathrm{D}_{3}$. In figs $3 \mathrm{c}$ and $3 \mathrm{~d}$ we see relatively weak anharmonic coupling between the infrared-active mode $\nu_{20}\left(E^{\prime}\right)$ and the Raman-active mode $\nu_{2}\left(A_{1}^{\prime}\right)$ of the $\mathrm{CH}$ fundamentals and the combination modes $\nu_{8}+\nu_{19}\left(\mathrm{~A}_{1}^{\prime}+\mathrm{A}_{2}^{\prime}+\mathrm{E}^{\prime}\right)$ and $v_{8}+v_{8}\left(\mathbf{A}_{1}^{\prime}+\mathbf{A}_{2}^{\prime}+E^{\prime}\right)$ [7]. Symmetry arguments allow the interaction of $\nu_{20}$ and $\nu_{2}$ with both combination modes, i.e. we have to consider four decay channels. Adding the different decay rates we finally estimate a lifetime of the $\mathrm{CH}$-stretching modes of $6 \mathrm{ps}$.

What do we find experumentally for the lifetime for the $\mathrm{CH}$-stretching modes? In fig. $4 \mathrm{~b}$ we present data on the population and depopulation of the $\nu_{2}$ mode at $3055 \mathrm{~cm}^{-1}$ during and after excitation of the molecule via the infrared-active $\nu_{20}$ vibration. The delayed maximum and the slope through the data points sug. gest a delay time $T_{1}=5 \pm 2 \mathrm{ps}$. This value is in good agreement with the estimates made above. It points 
Tablc 1

Summary of energy relexation tumes

\begin{tabular}{llcccc}
\hline Molecule & $\begin{array}{l}\text { Prequency } \mathrm{a}) \\
\left(\mathrm{cm}^{-1}\right)\end{array}$ & $N$ & $D$ & $\begin{array}{l}T_{1 \text { theor }} \\
\left(10^{-12} \mathrm{~s}\right)\end{array}$ & $\begin{array}{l}T_{1 \text { esp }} \\
\left(10^{-12} \mathrm{~s}\right)\end{array}$ \\
\hline $\mathrm{CH}_{2} \mathrm{CCl}_{2}$ & 3036 & 1 & 2 & 2 & $3 \pm 1$ \\
trans-CHClCHCl & 3073 & 2 & 1 & 13 & $10 \pm 2$ \\
$\mathrm{C}_{6} \mathrm{H}_{6}$ & 3063 & 5 & $>2$ & $<1$ & $1 \pm 05$ \\
$\mathrm{C}_{6} \mathrm{D}_{3} \mathrm{H}_{3}$ & 3055 & 3 & 1 & 6 & $5=2$ \\
\hline
\end{tabular}

a) Trequency of probed $\mathrm{CH}$-stretching modes, $N$ is the number of intually excited fundamental states tumes multuplicity. $D$ is the number of decay channels considered in the calculition, $T_{1}$ theor is the calculated lifeime, and $T_{1}$ esp is the cxpenmentally determined lifetıme.

to the importance of multiple decay channels for vibrathonal energy.

In table 1 the expenmental and calculated ufetumes are summarized for ready comparison.

In the preceding sections we have given arguments that the initally excited $\mathrm{CH}$-stretchung modes interact with well-defined overtones or combination modes. The question now arises whether we are able to see the population and depopulation in the subsequent energy states. There are several factors which determune the magnitude of the observable signal. (i) The vibrational mode of interest should have a large Raman scatterung cross section; (u) most of the excited energy should flow into one state, multiple decay chanuels are unfavorable; (w) the lifetume of the subsequent state should be equal or larger than the time constant of the primary level. In thus way the population in the following state accumulates for ready probing. Inspection of the infrared and Raman spectra in the neighborhood of the subsequent vibrations allows us to estimate anharmonic interaction with other modes. A prediction of the flow of energy to even lower states is thus possible.

As an example we discuss our observations of the molecule $\mathrm{CH}_{2}=\mathrm{CCl}_{2}$. We pointed out above that the two $\mathrm{CH}_{2}$-stretching modes decay va the combination modes $\nu_{2}+\nu_{3}$ and $\nu_{2}+\nu_{6}+\nu_{11}$. Both combination modes contain the infrared-and Raman-active $\mathrm{CH}$ bending mode at $\nu_{2}=1616 \mathrm{~cm}^{-1}$. We have searched for the anti-Stokes Raman signal of this bending mode and indeed found evidence for it. The scattered $\nu_{2}$ signal shufted at $1616 \mathrm{~cm}^{-1}$ has a maximum delayed by roughly 1 ps relative to the maximum of the $\nu_{1}$ curve of fig. 2a and the decay time was approximately equal to the $\nu_{1}$ data. Our observations suggest a short lifet ime of the $\nu_{2}$ level of $T_{1}<2$ ps. This estlmate is quite consistent with the infrared and Raman spectra of the $\nu_{2}$ mode where strong coupling with a nearby $\left(\omega \approx 50 \mathrm{~cm}^{-1}\right)$ overtone $2 \nu_{9}$ is very apparent As an additional decay route one has to consider the coupling to the $\nu_{3}$ fundamental which is only $\mathbf{2 2 0}$ $\mathrm{cm}^{-1}$ from the $\nu_{2}$ mode.

The results discussed in the preceding paragraph give interesting unformation on the decay of the $\nu_{2}$ mode. We do not know, however, whether we probe the combination state or the $\nu_{2}$ fundamental In the first case, the total vibrational energy of the molecule is $\approx 3000 \mathrm{~cm}^{-1}$, in the second case the total energy is $1616 \mathrm{~cm}^{-1}$ and the energy difference is resonantly transferred to ne,gliboring molecules.

\section{Conclusions}

Our find ings show convencingly that the population lifetime of vibrational modes of polyatomic molecules in the liquid phase depends - in a sensitive way - upon the individual vibrational energy states of the whole molecule. Snall changes of the synumetry of the molecule or of the frequency of vibrational states may lead to drastic changes of the lifetunes. Fermi resonances observed in unfrared and Raman spectra are a valuable gude for an estumate of the population lifetime.

\section{Acknowledgement}

The authors are indebted to Professor S.F. Fischer for many valuable discussions 


\section{References}

[1] R M Hoclistrasser, W. Kaser, C.V Shank, Picosccond phenomena, Vol 2 (Sprunger, Berlun, 1980).

[2] 4 Fendt, S.F. Fischer and W. Kaiser, Chem Phys 57 1981) 55

(3) ... Tendt, S F Fischer and W Kaser, Chem. Plyys. Letters 82 (1981) 350 .

[4] A Laubereau and W Kalser, Rev. Mlod Plys 50 (1978) 607
[5] G. Herzberg, Infrared and Ramian spectra of polyatomic molecules (Van Nostrand, Prunceton, 1945).

[6] S r Fischer, private communication.

[7] L M. Sverdlov, M A. Korvner and E.P. Krainov, Vibrational spectra of polyatomic molecules (Wilcy, New York, 1974), and references therein.

[8] C B Wuson Jr, J C Decius and P C. Cross, Molecular vibrations (McGraw-Hull, New York, 1955) p. 240 